\title{
3.4 Micro- and Macro-Vascular Remodeling and Cognitive Function in Hypertension
}

\author{
Antonio Gallo, ${ }^{1,2}$, Etienne Charpentier ${ }^{2}$, Thomas Dietenbeck ${ }^{2}$, Antoine Dufay ${ }^{1}$, Alban Redheuil ${ }^{3,4}$, \\ Marie Chupin ${ }^{2}$, Olivier Hanon ${ }^{7}$, Xavier Girerd ${ }^{1}$, Nadjia Kachenoura ${ }^{2}$
}

${ }^{1}$ University Hospital Pitié-Salpêtrière, Paris, France

${ }^{2}$ Sorbonne Universités, Paris, France

${ }^{3}$ Institute of Cardiometabolism and Nutrition (ICAN), Paris, France

${ }^{4}$ Pôle Imagerie-Groupe Hospitalier Pitié-Salpêtrière, Assistance Publique-Hôpitaux de Paris, Paris, France

${ }^{5}$ AP-HP, Hôpital Broca, Service de Gériatrie, Paris, France

\section{ABSTRACT}

Background: Cerebral microvascular alterations and hypoperfusion have been associated to cognitive disorder and dementia. We hypothesized that there is a direct link between retinal arteriolar remodelling, as assessed non-invasively using adaptive optics, and cognitive cerebral impairment in hypertensive patients.

Methods: Hypertensive patients over 65 years old with or without mild cognitive impairment were recruited. Each patient had a cognitive evaluation with MMSE; a study of retinal vasculature with adaptative optics RTX1 ${ }^{\oplus}$ Camera to measure WT, internal diameter (ID), wall-to-lumen ratio (WLR) and arterio-venous ratio (AVR); an aortic, cerebral and carotid MRI to study cerebral volumes and white matter hyper-intensities $(\mathrm{WMH})$ and carotid/aortic backward/forward flow (BF/FF) and distensibility. Correlations between retinal and cerebral, carotid and aortic remodelling were analysed.

Results: 51 treated and controlled hypertensive patients, aged of $74 \pm 5$ years, $67 \%$ males, were able to achieve all exams. Among subjects with higher WLR, a significantly lower MMSE was found $(p=0.04)$. Eye-Brain: AVR and arteriolar ID coefficient of variation were inversely related to cerebral volumes $(r=-0.61, p=0.01$ and $r=-0.58, p=0.02)$. Eye-Carotid: AVR and arteriolar ID coefficient of variation were directly and indirectly correlated with peak BF/FF, respectively $(r=0.47, p=0.005 ; r=-0.39$, $p=0.02)$. Eye-Aorta: WT and AVR were inversely correlated with ascending aorta distensibility $(r=-0.48, p=0.003 ; r=-0.47$, $p=0.007)$

Conclusion: In elderly hypertensives, arteriolar retinal remodelling represents a potential marker of early cerebral atrophy, which might be linked to a cerebral microcirculation remodelling. This hypothesis is supported by the association between retinal vascular remodelling and impaired carotid flow and aortic elastic properties.

(c) 2019 Association for Research into Arterial Structure and Physiology. Publishing services by Atlantis Press International B.V. This is an open access article distributed under the CC BY-NC 4.0 license (http://creativecommons.org/licenses/by-nc/4.0/). 